\title{
Reference Model as a Factor of Quality Improvement of Management Under Uncertainty
}

\author{
Emelyanov A.E.* \\ Voronezh State University of Engineering Technologies \\ Voronezh, Russia \\ e-mail: emalexeg@yandex.ru
}

\author{
Bityukov V.K. \\ Voronezh State University of Engineering Technologies \\ Voronezh, Russia
}

\begin{abstract}
The subject of the research is to study the improvement of the quality of the management process, which is inextricably linked with the digitalization of management activities. The purpose of this work is to develop a simulation model that includes a reference model of an object, which allows analyzing the quality of control of various systems with probabilistic loss of information about the state of the control object. Computer simulation of control systems with a network channel for transmitting information was carried out for various probabilities of loss of information from the measuring device. It was assumed that the transmission of information over a network channel during the quantization period is carried out with a given probability. If the data packet from the measuring device is not transmitted in one quantization cycle to the control device, then it is considered to be lost. The control device processed the incoming information sequentially. It is shown that the developed simulation model allows analyzing the quality of transients of control systems using network stochastic information transmission channels. The examples considered show that a network management system with low probabilities of information loss provides a satisfactory quality of the transition process. However, with an increase in the probability of information loss, the control system without using the reference model of the control object significantly worsens the quality indicators of the control process and may even lose stability. The study showed that the use of a reference model of the control object significantly improves the quality of transients. The innovation of the developed simulation model is in the fact that its development is based on modeling both the probability of losing information about the state of the control object and the reference model of the object itself. Modeling the functioning of the network control system was carried out in the Simulink environment of the Matlab system.
\end{abstract}

Keywords - quality of control, network system, reference model, simulation model, probability, information

\section{INTRODUCTION}

The improvement of the quality of the management process in various systems is inextricably linked with the digitalization of management, which is the basis for the creation of innovative technologies. The introduction of innovations involves the implementation of the pairing of maintenance personnel, equipment, regulatory, measuring and information systems by eliminating technical contradictions between the functioning and the control of the process flow.

The optimization of the control process in various systems requires taking into account the dynamics of changes in the parameters of the control process on the entire planning horizon. The problem of analysis and synthesis of the structure of the organizational management system includes the choice of the number of levels and subsystems, as well as the principles of management organization; synthesis of the structure of the system for collecting, transmitting and processing information; resolving issues related to the optimization of the hierarchy of creating a system that ensures the effective implementation of management functions and the achievement of goals. For this, methods of information theory and control theory are increasingly being used [1]. Moreover, the uncertainty and risk of loss of information about the current state of management facilities are an integral part of any management activity [2]. Loss of information occurs, as a rule, due to unreliable channels for obtaining information. In technical systems, channels of this kind are called network channels, and systems are called Networked Control System (NCS) [3, 4]. In the future, we will adhere to this terminology. Such data transmission channels have a number of features random delays and loss of information during transmission from one system element to another [5-10]. It is shown in the literature that random delays and loss of information have a significant impact on the quality of control of various systems.

In order to analyze the modes and quality of control in systems using network channels for transmitting information, simulation modeling is used. This requires the use of methods and approaches, both control theory and communication theory. This fact significantly complicates the analysis, modeling and synthesis of such control systems [11-13]

In $[14,15]$, the analysis of information transmission time in distributed systems with competing access to the network channel was carried out. The results of the analysis showed that the transmission time of information can be satisfactorily described by the exponential distribution law. In [14, 15], an attempt was made to compensate the loss of information by duplicating it. However, the influence of the amount of duplicated information on the quality of system management has not been considered.

In this paper, to improve the quality of control with uncertainty in obtaining information about the current state of the control object, it is proposed to use the reference model of the object in the control loop.

The purpose of this work is to elaborate a simulation model that includes a reference model of an object, which allows analyzing the quality of control of various systems with probabilistic loss of information about the state of the control object. 
The innovation of the developed simulation model is in the fact that its development is based on modeling both the probability of losing information about the state of the control object and the reference model of the object itself.

\section{METHODS AND MATERIALS}

Modeling the functioning of the network control system was carried out in the Simulink environment of the Matlab system. The developed scheme (Fig. 1) of the control system uses a network channel for transmitting information between the measuring device and the control device.
This system works as follows. The measuring device receives information about the regulatory object at certain points in time and transfers it further to the control device via a network channel. In this case, a network channel with a certain probability can be in one of two states: "open", when information transmission through it is possible, and "closed", when information transmission through it is impossible. If the channel is "open", then the measuring device immediately transmits the received information to the control device. If the channel is "closed", then information from the measuring device is considered to be lost for the control device.

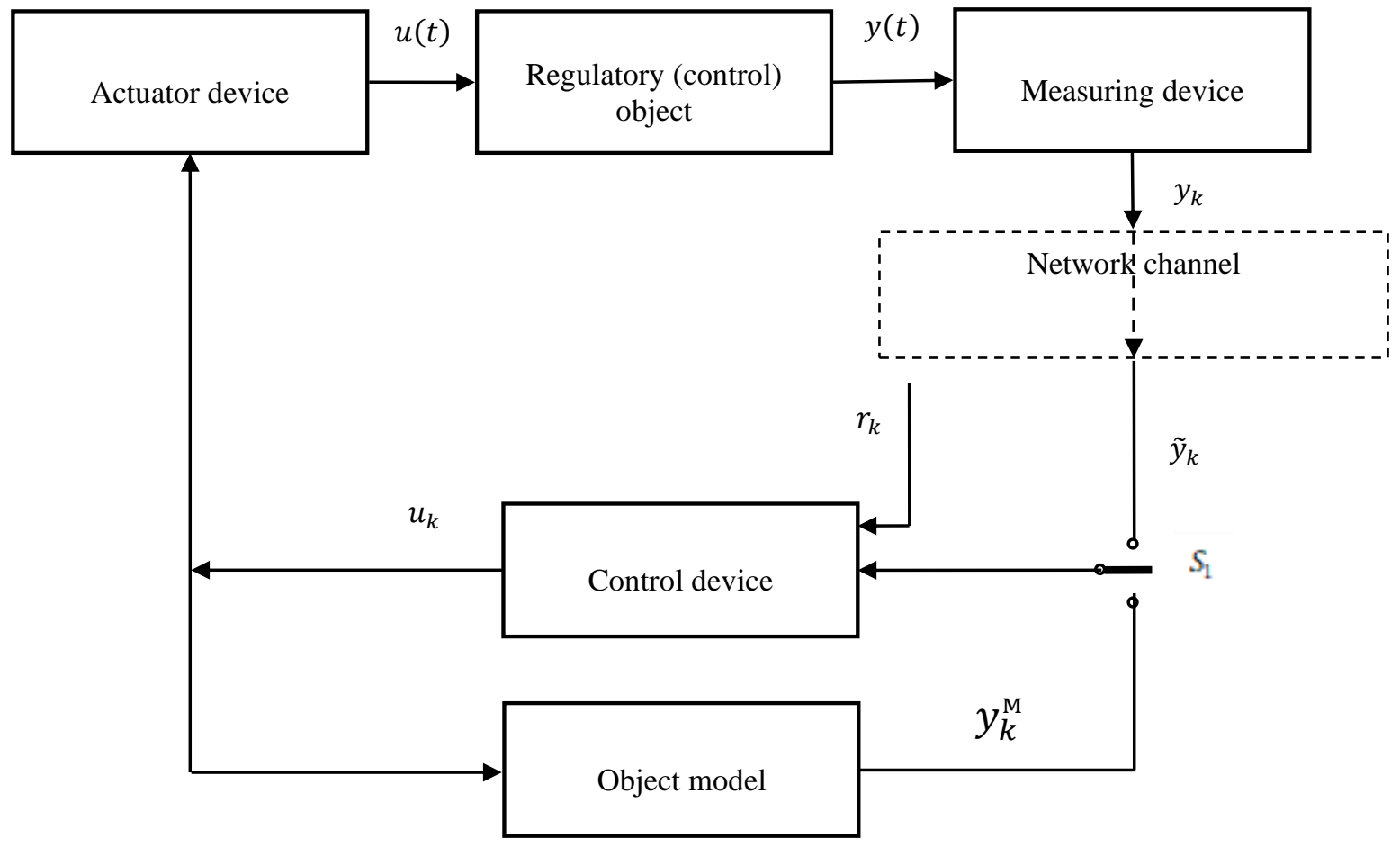

Fig. 1. Control system with a reference model of the control object

The control device sequentially processes the incoming information from the measuring device. The control device generates a control action $u_{k}$ according to a specific, predetermined algorithm, taking into account the set action $r_{k}$ and the received information from measuring device $y_{k}$. The regulatory action $u_{k}$ is transmitted to the actuator at time $t=k T_{0}$. The control action of the actuator is implemented at the control object $u(t)$.

It is assumed that the measuring device and the control device operate synchronously: information is received by the measuring device and control action is generated by the control device at the same time intervals (quantization cycles) $T_{0}$ and at the same time $t=k T_{0}$.

It is assumed that the characteristics of the measuring device and actuator do not influence on the control process of the system. Therefore, it was assumed that the measuring device and actuator correspond to inertia less elements with a unity gain.
The control object was modeled by the serial connection of the inertial link of the first order and the integral link. The control device produced the control action according to the proportional-integral-differential algorithm.

To improve the quality of management, a reference model of the control object is included in the decision-making system.

According to the reference model of the control object, information $y_{k}^{\mathrm{M}}$ is calculated for each moment in time $t=k T_{0}$. If the information from the measuring device is transmitted over the network channel to the control device in time $T_{0}$, this information will be taken into account when developing a new control action. If, in time $T_{0}$, information from the measuring device is not transmitted to the control device, then when developing the control action, information from the reference model of the control object will be used. In fig. 01 is shown a key $S_{1}$ that implements this algorithm for accounting information from a measuring device. Information from the measuring device that has not been transmitted during the time $T_{0}$ is considered to be lost. 
The simulation scheme of the control system is in Fig. 2. This scheme simulates the behavior of the system that is described above. While modeling, it was assumed that the model exactly corresponds to the control object. The key algorithm $S_{1}$ in this scheme is implemented using the switch Switch 1.

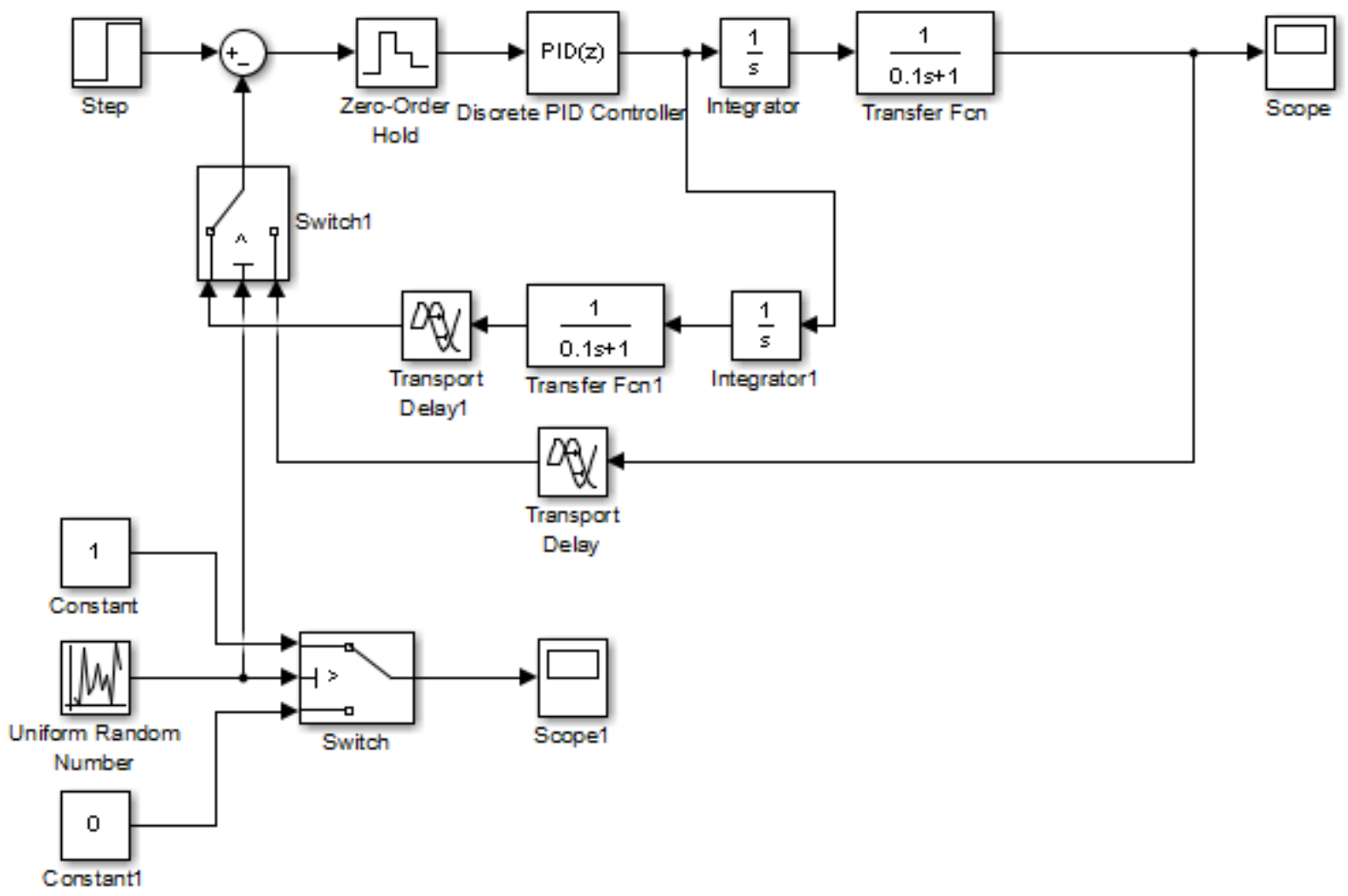

Fig. 2. The simulation scheme of the control system with a reference model of the control object

\section{RESULTS}

The numerical results of the studies are presented in the form of graphs of transient processes (Fig. 3-7).

Here: $P$ is the probability of channel "closure"; $T_{0}$ is the quantization cycle.
In fig. 3-6, the control system operates without a reference model.

As it can be seen from the analysis of the transients, the system functions satisfactorily with a low $(P=0.2)$ and medium $(P=0.4)$ probability of information loss (Fig. 3, 4).

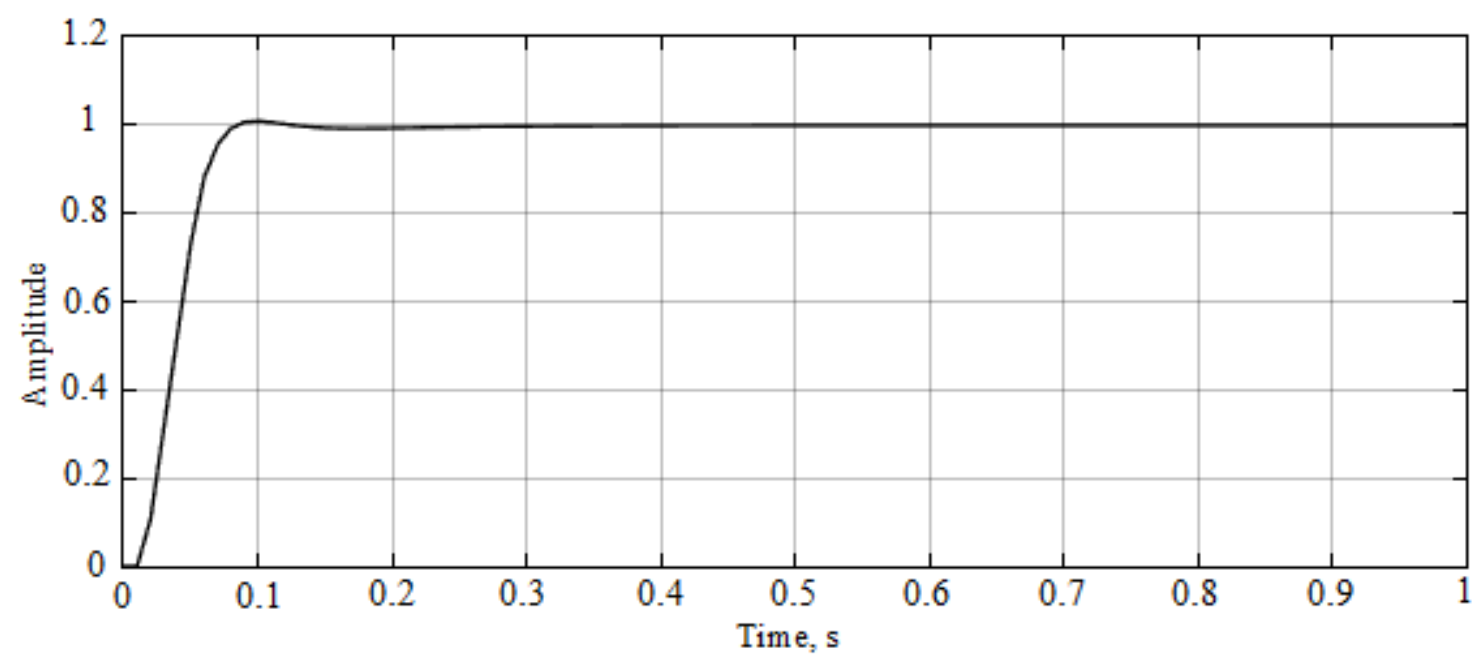

Fig. 3. Transient process: $\mathrm{P}=0.2 ; T_{0}=0.01$ 
However, a further increase in the probability of loss of information $(P=0.8)$ about the control object significantly worsens the quality indicators of the control process (Fig. 5): overshoot approaches $50 \%$, the control time increases from 0.1 to $0.3 \mathrm{~s}$, and the transient oscillation increases significantly.

Especially the deterioration in the quality of the transient process with a further increase in the probability of information loss $(P=0.9)$ is seen in Fig. 6. The transition process is close to an unstable regime.
In fig. 7 shows the transition process of the control system, with the probability of loss of information $P=0.9$. From the analysis of the transition process, we can conclude that the control system is stable, quality management indicators are satisfactory: there is no overshoot. The transition process time is $0.1 \mathrm{~s}$.

It should be noted that the transition process to a step change in the set action corresponds to a network control system with a delay in the feedback control loop by one quantization cycle $T_{0}$.

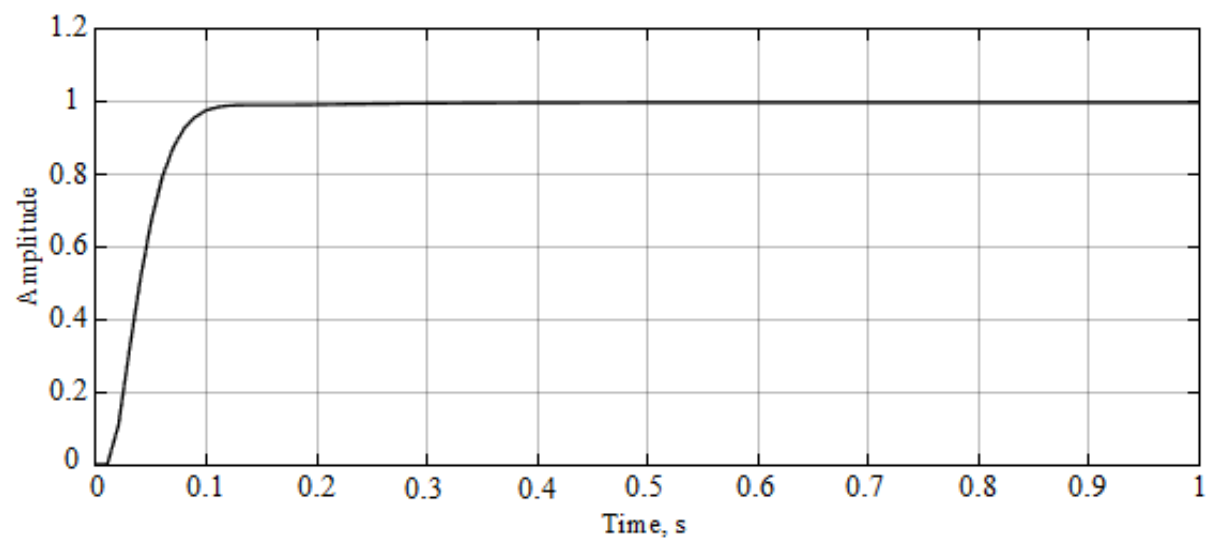

Fig. 4. Transient process: $\mathrm{P}=0.4 ; T_{0}=0.01$

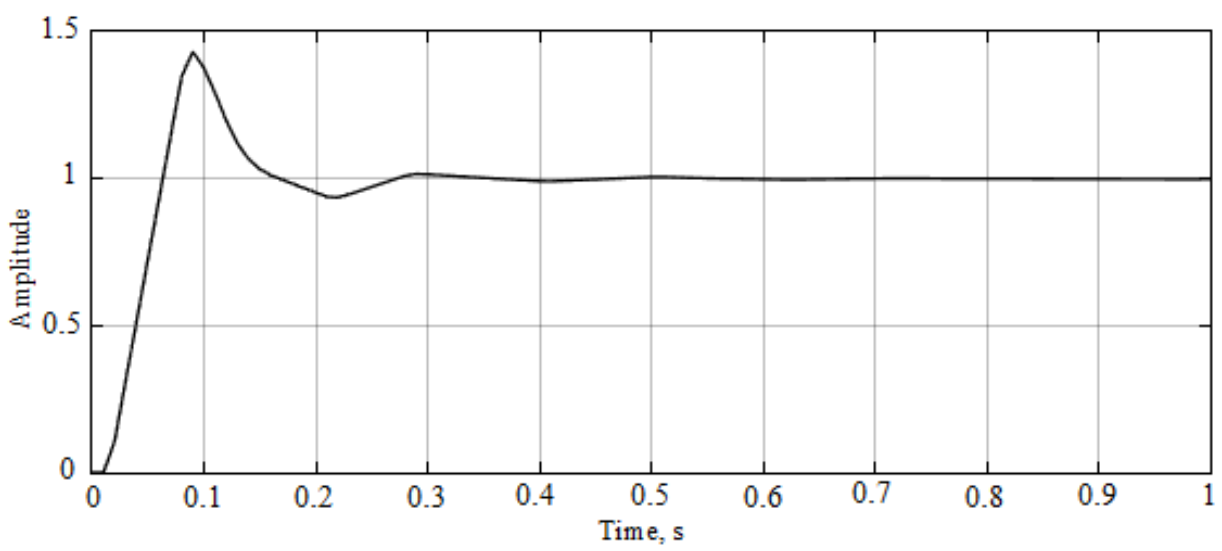

Fig. 5. Transient process: $\mathrm{P}=0.8 ; T_{0}=0.01$

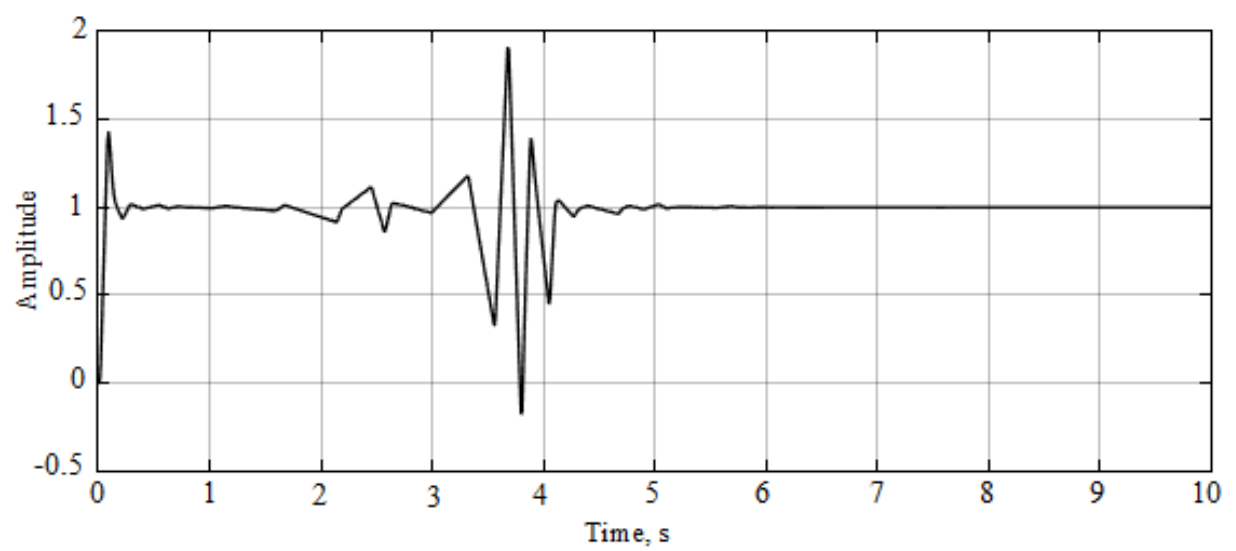

Fig. 6. Transient process: $\mathrm{P}=0.9 ; T_{0}=0.01$ 


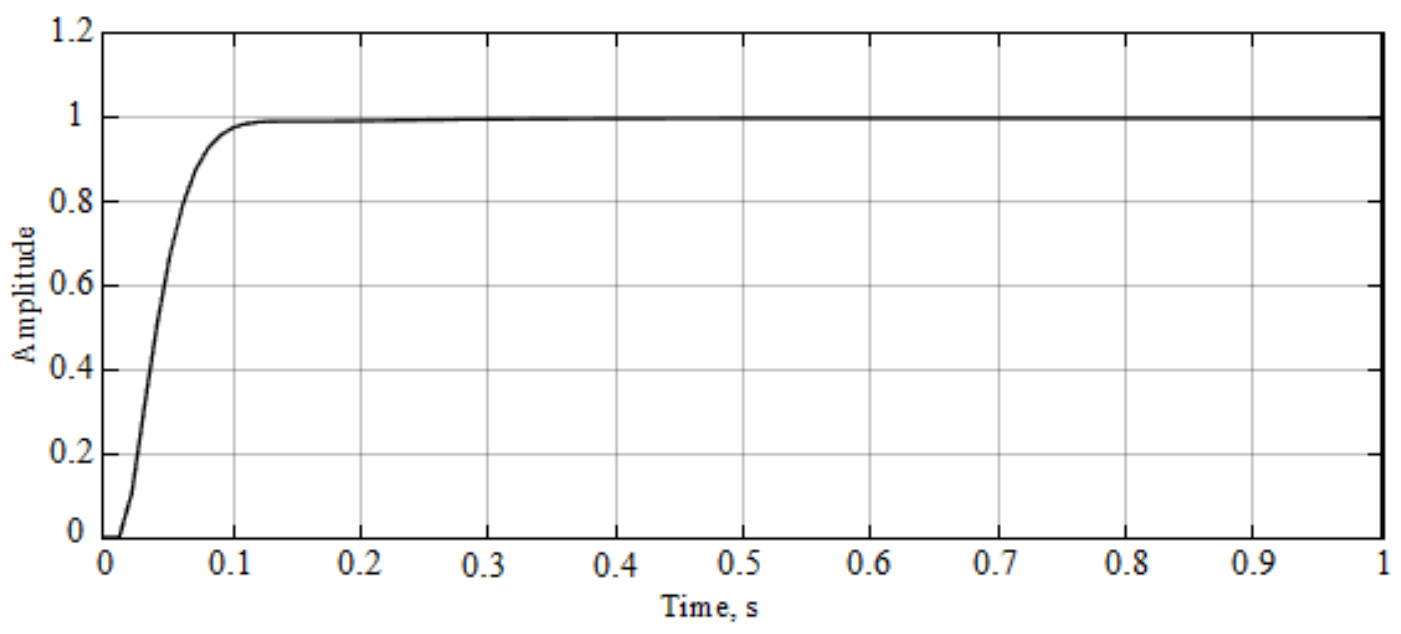

Fig. 7. Transient process: $\mathrm{P}=0.9 ; T_{0}=0.01$

\section{CONCLUSION}

The developed simulation model allows analyzing the quality of transient processes of control systems using network stochastic information transmission channels. The considered examples show that a network management system with low probabilities of information loss provides a satisfactory quality of the transient process. However, with an increase of the probability of the information loss, the control system without using the reference model of the control object significantly worsens the quality indicators of the control process and may even lose stability. The study showed that the use of a reference model of the control object significantly improves the quality of transient processes.

The developed simulation model is based on modeling both the probability of loss of information about the state of the control object and the reference model of the object itself.

Compared with the known approaches of mathematical modeling of network control systems [16], the obtained model allows evaluating the efficiency of using the existing model of the object as a reference.

The practical value of the developed simulation model is that it can be used in the design of new network control systems, as well as in the modernization of systems that are already used in practice.

\section{References}

[1] A.N. Desyatirikov, Y.B. Nechaev, E.N. Desyatirikova, A.Y. SHestopalov, "Information analysis and synthesis of the organizational structure of the unique project in the classical control theory", Proc. of the Voronezh State Univer. of Engineer. Technol., vol. 80, no. 4, pp. 463-470, 2018.

[2] T.V. Sabetova, "Identification and evaluation of the impact of uncertainty factors on the activities of agricultural enterprises", Proc, of the Voronezh State Univer, of Engineer, Technol,, vol. 80, no. 4, pp. 417-425, 2018.

[3] H. Wu, "Cloud-based net- worked visual servo control", IEEE Transact. on Industrial Electronics, vol. 60, p. 554, 2013.

[4] L. Zhang, "Network-induced constraints in networked control systems a survey", IEEE Transactions on Industrial Inform., vol. 9, p. 403, 2013.
[5] M-D. Hua, T. Hamel, P. Morin, C. Samson, "Introduction to feedback control of underactuated VTOL vehicles: a review of basic control design ideas and principles", IEEE Control Syst., vol. 33, no. 1, p. 61, 2013.

[6] K-Y. You, L-H. Xie, "Survey of recent progress in networked control systems", Sci. China Inf. Sci., vol. 39, no. 2, p. 101, 2013.

[7] X. Chen, F. Hao, "Periodic event-triggered state-feedback and outputfeedback control for linear systems", Int. J. Control Autom. Syst., vol. 13, no. 4, p. 779, 2015.

[8] O.Ja. Kravets, O.N. Choporov, "The problems and peculiarities of modelling integreted systems of heterogeneous traffic service", J. Siber. federal Univer., Ser. Mathem. and phys., vol. 11, no. 5, pp. 581-587, 2018.

[9] V. Povarov, A. Danilov, V. Burkovsky, K. Gusev, "Data support system for controlling decentralised nuclear power industry facilities through uninterruptible condition monitoring", MATEC Web of Conf., vol. 161, 2018.

[10] T. Gommans, D. Antunes, T. Donkers, P. Tabuada, W. Heemels, "Selftriggered linear quadratic control", Automatica, vol. 50, no. 4, p. 1279, 2014.

[11] O.Ya. Kravets, O.N. Choporov, V.E. Bolnokin, "Mathematical models and algorithmization of monitoring control an affiliated network in maintenance service distributed organizations", Quality - Access to Success, vol. 19, no. 167, pp. 68-72, 2018.

[12] M.A. Kutsakin, A.N. Lapko, E.V. Lebedenko, V.V. Ryabokon, "To the issue of verification of the adequacy of a simulation model system of decentralized management process of network planning based on intelligent autonomous agents", Inform. Syst. and technol.s, vol. 1, no. 111 , pp. 30-36, 2019.

[13] E.V. Lebedenko, A.A. Minaichev, "Model of processing system of the multi-service data high-speed backbone channels with non-stationary load", Telecommunicat., vol. 8, pp. 27-29, 2017.

[14] G.V. Abramov, A.E. Emelyanov, K.Ch. Kolbaya, "Analysis of the transmission time of data in distributed networks with competing access", Vest. of the Voronezh State Univer., vol. 4, pp. 61-67, 2016.

[15] G.V. Abramov, A.E Emelyanov, A.L Ivashin, "Identification of applicability area of mathematical model of network control system functioning in asynchronous mode during data transfer via multiple access channel, vol. 3, pp. 199-202, 2011 [The 15th World Multi-Conf. on Systemics, Cybernetics and Informatics, Proceedings (WMSCI 2011)].

[16] D. Huang, S.K. Nguang, "Robust Control for Uncertain Networked Control and Information Sciences, Delays", Ser. Lecture Notes in Control and Inform. Sci., vol. 38, p. 146, 2009 . 